\title{
Quaternary epitope landscape of Zika virus antibody complexes
}

\author{
Madhumati Sevvana and Richard Kuhn \\ Purdue University, West Lafayette, Indiana, United States
}

\begin{abstract}
Zika virus (ZIKV), a mosquito-borne human flavivirus is closely related to dengue virus, yellow fever virus, West Nile virus and Japanese encephalitis virus. ZIKV can cause congenital Zika syndrome in infants and Guillain-Barré syndrome in adults. Neutralizing antibodies (nAbs) are amongst the preferred antiviral therapeutic strategies against infection by flaviviruses. Successful vaccines exist against YFV, JEV and TBEV. However, vaccine development against other flaviviruses like dengue virus is not straightforward. This is partly because of the high sequence conservation and immunological crossreactivity among flavivirus envelope glycoproteins leading to antibody mediated enhancement of disease (ADE). Thus, understanding the immune response in consecutive flavivirus infections and virus neutralization mechanisms by various classes of nAbs may help to prevent disease severities leading to $\mathrm{ADE}$, which is a major risk factor for vaccine development.

The structures of the mature ${ }^{1-3}$ (Figure 1A) and the immature ${ }^{4}$ ZIKV and its complexes with nAbs, unfolded structural components important for infection and the binding epitopes for diverse nAbs. In summary, the nucleocapsid core formed by the capsid protein-RNA complex in a mature virus is protected by the heterodimeric arrangement of the glycoproteins, E-M, embedded in a host-derived lipid envelope. The E protein, in addition to its role in virus assembly contains putative receptor binding sites and mediates virus entry by $\mathrm{pH}$ mediated fusion with the host membrane. The E ectodomain $\left(\mathrm{E}_{\mathrm{e}}\right)$ accessible to nAbs, consists of three domains: a central $\beta$-barrel domain I (DI), which connects to the dimerization domain II (DII), and an Ig-like receptor binding domain III (DIII) (Figure 1A). E protein DII harbors a conserved hydrophobic sequence, called the fusion loop (FL), a feature of type-II fusion proteins. The FL inserts into the host cell membrane during $\mathrm{pH}$-induced conformational changes leading to virus-host membrane fusion during infection. Several ZIKV-nAb complexes have been structurally and functionally characterized ${ }^{5,6}$. The nAbs against $\mathrm{E}$ might inhibit several processes during infection including receptor binding, blocking conformational changes and exposure of the FL. Among these, the primary focus will be on structural aspects of ZIKV nAbs utilizing novel quaternary epitopes on the virus surface (Figure 1B and 1C). Quaternary epitopes span over multiple E proteins decorating the surface of the virus or multiple domains (DI-II-III) of more than one E protein. The nAbs that will be discussed here were identified either in individuals with subsequent flavivirus infections or discovered as a result of a sprint program to deploy protective antibody treatments against viral outbreaks. The binding stoichiometry and neutralization mechanisms conferring high potency and virus specificity of the nAbs with quaternary epitopes will be discussed and the landscape of the quaternary epitopes among diverse flaviviruses will be compared.
\end{abstract}

\section{Acknowledgements}

The results presented here are in collaboration with the groups of Dr. Dennis Burton (Scripps Institute), Dr. James Crow Jr (Vanderbilt Vaccine Center) and Dr. Robert Carnahan (Vanderbilt University School of Medicine). All authors are not included because of space restrictions. We thank Michael G. Rossmann, who was the inspiration and intellectual powerhouse for many of the flavivirus structural studies. 
(A)

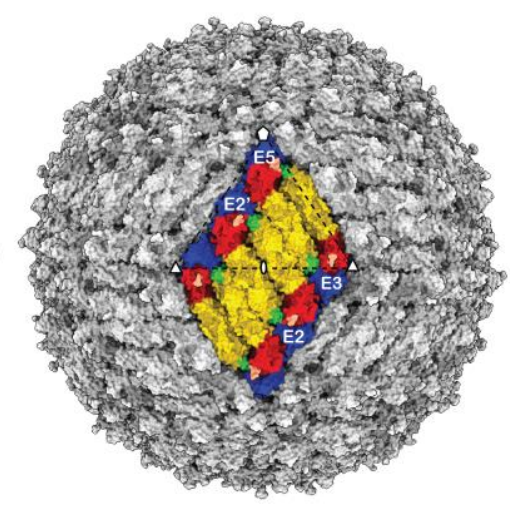

(B)

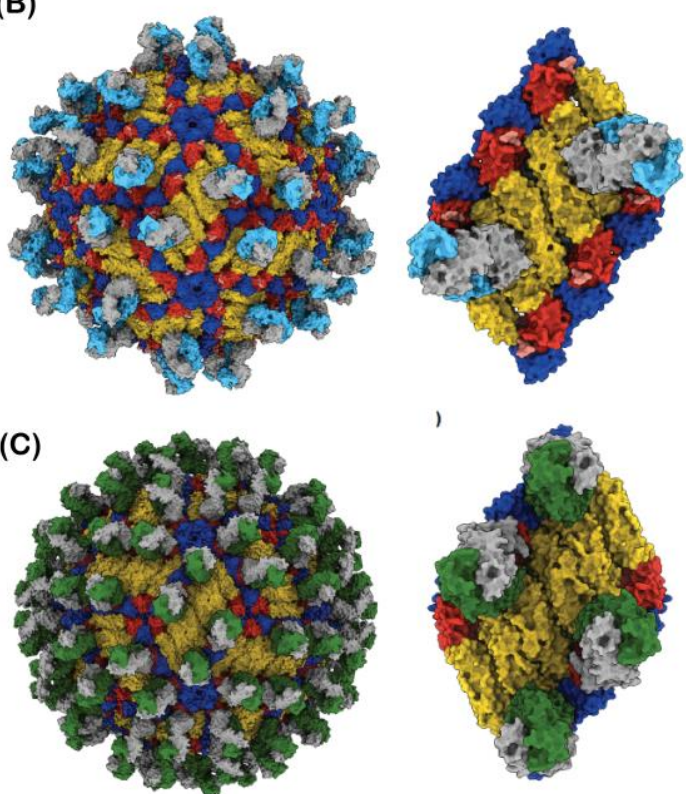

Figure 1. (A) ZIKV structure showing the herringbone pattern formed by $6 \mathrm{E}-\mathrm{M}$ heterodimers. One icosahedral asymmetric unit is identified by a black triangle. For clarity only the ectodomain of the $\mathrm{E}$ protein (from residues 1-400) has been shown. The E proteins near the two-fold, three-fold and five-fold axes of symmetry are labelled E2, E3 and E5, respectively. Domains E-DI, E-DII and E-DIII are colored red, yellow and blue, respectively. (B-C) Representative structures of ZIKV-nAb complexes with quaternary epitope. (B) E-protein Domain-II specific antibody and (C) ZIKV in complex with envelope protein Domain-I-II specific antibody.

\section{References}

(1) Hasan, S. S., Sevvana, M., R. J. Kuhn and M. G. Rossmann (2018). Nat Struct Mol Biol. 25, 13-20.

(2) Sirohi, D., Z. Chen, L. Sun, T. Klose, T. C. Pierson, M. G. Rossmann and R. J. Kuhn (2016). Science 352: 467-470.

(3) Sevvana M, Long F, Miller AS, Klose T, Buda G, Sun L, Kuhn RJ, Rossmann MG. Refinement and Analysis of the Mature Zika Virus Cryo-EM Structure at $3.1 \AA$ Resolution. Structure. 2018 Sep 4;26(9):1169-1177.

(4) Prasad, V. M., A. S. Miller, T. Klose, D. Sirohi, G. Buda, W. Jiang, R. J. Kuhn and M. G. Rossmann (2017). Nat Struct Mol Biol. 24: 184-186

(5) Sevvana, M. and Kuhn. R. J. The diverse structural landscape of antibody repertoire against flaviviruses. (2020). Current Opinions in Virology.

(6) Sevvana, M., Rogers, TR., Miller, AS., Long, F., Beutler, N., Lai, YC., Parren, M., Walker, LM., Buda, G., Klose, T., Burton, DR., Rossmann, MG., Kuhn, R. J. Structural perspectives on Zika virus specific neutralization in subsequent flavivirus infections. (2020). Viruses. 